\title{
Einfluss der Alterung UF-Harz gebundener Holzspanplatten auf die Abgabe flüchtiger Säuren
}

\author{
Edmone Roffael $\cdot$ Mahmood Hameed $\cdot$ Claus Behn
}

Published online: 29 November 2008

(C) Die Autoren 2008. Dieser Artikel wurde mit Open Access auf Springerlink.com veröffentlicht.

\begin{abstract}
Zusammenfassung Die Abgabe an flüchtigen Säuren von UF-Harz gebundenen Kiefernholzspanplatten wurde nach der Herstellung sowie nach einer Alterungsdauer von 13 Jahren bestimmt. Die Ergebnisse lassen erkennen, dass die Abgabe an Ameisensäure durch die Alterung der Platten deutlich abgenommen hat, während die Abgabe an Essigsäure signifikant zugenommen hat. Auf diesen Zusammenhang scheint der bei der Plattenherstellung verwendete Härtertyp (Ammoniumnitrat, Ammoniumchlorid) und die eingesetzte Härtermenge keinen entscheidenden Einfluss zu nehmen.
\end{abstract}

\section{Influence of aging of UF-bonded particleboards on the release of volatile organic acids}

\begin{abstract}
The release of volatile organic acids from UFbonded particleboards made from Scots pine was assessed after production as well as after aging for 13 years. The results reveal that aging significantly decreased the release of formic acid and substantially increased the emanation of acetic acid. The change in the release of volatile acids by
\end{abstract}

E. Roffael (汭)

Georg-August-Universität Göttingen,

Büsgenweg 4,

37077 Göttingen, Deutschland

e-mail: eroffae1@gwdg.de

M. Hameed

Universität Damaskus,

P.O. Box 29082, Damascus, Syria

C. Behn

Büsgen-Institut, Abteilung Molekulare Holzbiotechnologie und Technische Mykologie, Georg-August-Universität Göttingen, Büsgenweg 2,

37077 Göttingen, Deutschland aging seems to be highly independent of the hardener type used (ammonium chloride, ammonium nitrate) as well as of the amount of added hardener.

\section{Einleitung}

Es ist weithin bekannt, dass nach dem Pressvorgang in Holzspan- und -faserplatten immer noch chemische Reaktionen ablaufen, die das chemische und physikalische Verhalten der Platten deutlich beeinflussen. So ist es bewiesen, dass mit alkalisch härtenden Phenol-Formaldehydharzen (PF-Harzen) hergestellte Holzspanplatten durch Stapelung und Lagerung an Biege- und Querzugfestigkeit deutlich zunehmen und an Dickenquellung im Wasser erheblich abnehmen (vgl. Roffael et al. 1988). Dies wird auf eine Nachkondensation der Phenol-Formaldehydharze während der Stapelung und Lagerung zurückgeführt. Die Vergütung der PF-gebundenen Holzspanplatten durch die Stapelung und Lagerung wird auch von einer Verringerung der ohnehin sehr niedrigen Formaldehydabgabe der PF-gebundenen Sperrholz- und Holzspanplatten begleitet (vgl. Emery 1986). Auch im Falle von UF-gebundenen Holzspan- und Holzfaserplatten nimmt die Emission an Formaldehyd durch die Alterung signifikant ab (vgl. Sundin u. Roffael 1989). Auch eine gewisse jedoch nicht stets ausgeprägte Abnahme der Dickenquellung wurde festgestellt. Auf die Ursache der Formaldehydabgabeverminderung durch Alterung sind eingehende Forschungsarbeiten, soweit bekannt, bislang nicht systematisch angestellt worden. Es wird jedoch angenommen, dass Formaldehyd im Zuge der Alterung mit dem UF-Harz vernetzend reagiert, dies ist insofern vorstellbar als der pH-Wert in Holzspanund Holzfaserplatten nach der Herstellung im saueren Bereich liegt und zudem die Holzspanplatten und Holzfaser- 
platten immer noch freien Harnstoff enthalten (Hameed et al. 2006). Reaktionen zwischen Formaldehyd und Holz in saurer Umgebung sind ebenfalls denkbar. Während über die Veränderung der Formaldehydabgabe von Holzwerkstoffen mit der Alterung bereits umfangreiches Schrifttum existiert (vgl. Çolakoğlu u. Roffael 1991), liegen, soweit bekannt, über die Veränderung der Emission an flüchtigen Säuren wie Ameisen- und Essigsäure in Abhängigkeit von der Alterung keine Ergebnisse von systematischen Untersuchungen vor. Ameisen- und Essigsäure entstehen auch durch die thermischen und chemischen Prozesse im Holz bei der Herstellung von Holzspan- und Holzfaserplatten. So kann sich Ameisensäure durch Abbau der Kohlenhydrate, insbesondere die Pentosanen, während der Trocknung der Späne bei der Spanplattenherstellung oder während des Aufschlusses von Hackschnitzeln im Zuge der Faserplattenherstellung bilden. Untersuchungen in dieser Richtung sind insofern von Interesse, als UF-Harz-Systeme durch die allgemeine Säure-Base-Katalyse kontrolliert sind (Petersen et al. 1972) und oftmals auch Ameisensäure als Härtungsbeschleuniger in Aminoplastharzsystemen herangezogen wird.

\section{Material und Methoden}

Als Versuchsmaterial dienten UF-Harz gebundene Kiefernholzspanplatten, für deren Herstellung sowohl Ammoniumnitrat als auch Ammoniumchlorid in unterschiedlichen Mengen $(0,8,1,6$ und 2,4\% Feststoff bezogen auf Feststoffbindemittel) als Härtungsbeschleuniger verwendet wurden. Die Eigenschaften der verwendeten Harnstoffharze

Tabelle 1 Eigenschaften der verwendeten Harnstoffharze Table 1 Properties of UF-resins used

\begin{tabular}{lc}
\hline Eigenschaft & UF-Harz \\
\hline Feststoffgehalt (\%) & 65,2 \\
pH-Wert & 8,23 \\
Stickstoffgehalt (\%) & 23,1 \\
Molverhältnis F : H & $1,12: 1$ \\
Freier Formaldehydgehalt (\%) & 0,09 \\
Gesamtformaldehydgehalt (\%) & 27,7 \\
Viskosität (50\%iges Harz) & 46 \\
D = 1000 s ${ }^{-1}$ (mPa.s) & \\
Wasserverträglichkeit & $1: 1,18$ \\
UF-Harz : Wasser & \\
\hline
\end{tabular}

Tabelle 2 Die Bedingungen für die Herstellung der untersuchten Spanplatten (nach Lee et al. 1994)

Table 2 Conditions of particleboard preparation (Lee et al. 1994)

\begin{tabular}{ll}
\hline Abmessungen & $\begin{array}{l}570 \mathrm{~mm} \times 510 \mathrm{~mm} \\
(510 \mathrm{~mm} \times 460 \mathrm{~mm} \text { besäumt })\end{array}$ \\
Solldicke & $20,5 \mathrm{~mm}(19 \mathrm{~mm}$ nach dem Schleifen) \\
Bindemitteltyp & UF-Harz (vgl. Tabelle 1) \\
Bindemittelgehalt & $8 \%$ \\
Feuchte der beleimten Späne & $9,5-10,5 \%$ \\
Presstemperatur & $190^{\circ} \mathrm{C}$ \\
Presszeit & $15 \mathrm{~s} / \mathrm{mm}$ \\
Hydrophobierungsmittel & Ohne Zusatz \\
Härtungsbeschleuniger & Ammoniumnitrat (AN), Ammonium- \\
& chlorid (AC) $(0,8,1,6,2,4 \%)$ \\
\hline
\end{tabular}

sind in Tabelle 1 aufgeführt. Diese Spanplatten wurden im Jahr 1993 unter den in Tabelle 2 aufgeführten Bedingungen hergestellt (Lee et al. 1994). An diesen Spanplatten wurde nach der Herstellung sowie nach einer Lagerungsdauer von 13 Jahren von Herstellungsdatum an gerechnet die Abgabe an Ameisen- und Essigsäure bestimmt. An den Proben wurden des Weiteren folgende Platteneigenschaften ermittelt:

- Feuchtegehalt (EN 322) (Tabelle 3),

- pH-Wert von Kaltwasser-Extrakten (Tabelle 3),

- Säureabgabe (Ameisen- und Essigsäure 24, 48 h) nach der WKI-Flaschen-Methode (vgl. Roffael 1982, Roffael 1989).

\section{Ergebnisse und Diskussion}

Die Ergebnisse über die Abgabe an Ameisen- und Essigsäure sind in Abb. 1, 2, 3 und 4 zusammengestellt. Diese lassen erkennen, dass die Abgabe an Ameisensäure durch die Alterung der Platten, unabhängig von dem eingesetzten Härtungsbeschleuniger, erheblich abgenommen hat. Auch die Menge an eingesetztem Härter scheint für diesen Trend keine gewichtige Rolle zu spielen. So lagen die Abgabewerte der für 13 Jahre unter ambienten Bedingungen gelagerten Holzspanplatten mehr oder minder um etwa 40-50\% niedriger als vorher. Dies ist nach einer Prüfdauer von 24 als auch $48 \mathrm{~h}$ im Flaschentest deutlich festzustellen. Inwieweit die Abnahme der Emission an Ameisensäure mit der Verringerung der Formaldehyd-
Tabelle 3 Feuchtegehalt und pH-Werte von KaltwasserExtrakten der UF-Harz gebundenen Kiefernholzspanplatten

Table 3 Moisture content and $\mathrm{pH}$-value of particleboards bonded with UF-resin

\begin{tabular}{lcccccc}
\hline Plattentyp & I & II & III & IV & V & VI \\
\hline Feuchtegehalt (\%) & 8,24 & 8,28 & 8,28 & 8,29 & 7,98 & 8,20 \\
pH-Wert & n.b. & 4,71 & 4,65 & n.b. & 4,67 & 4,59 \\
Bindemitteltyp & UF & UF & UF & UF & UF & UF \\
Härter (\%) & AN 0,8 & AN 1,6 & AN 2,4 & AC 0,8 & AC 1,6 & AC 2,4 \\
\hline
\end{tabular}

(AN: Ammoniumnitrat; AC: Ammoniumchlorid, n.b.: nicht bestimmt) 


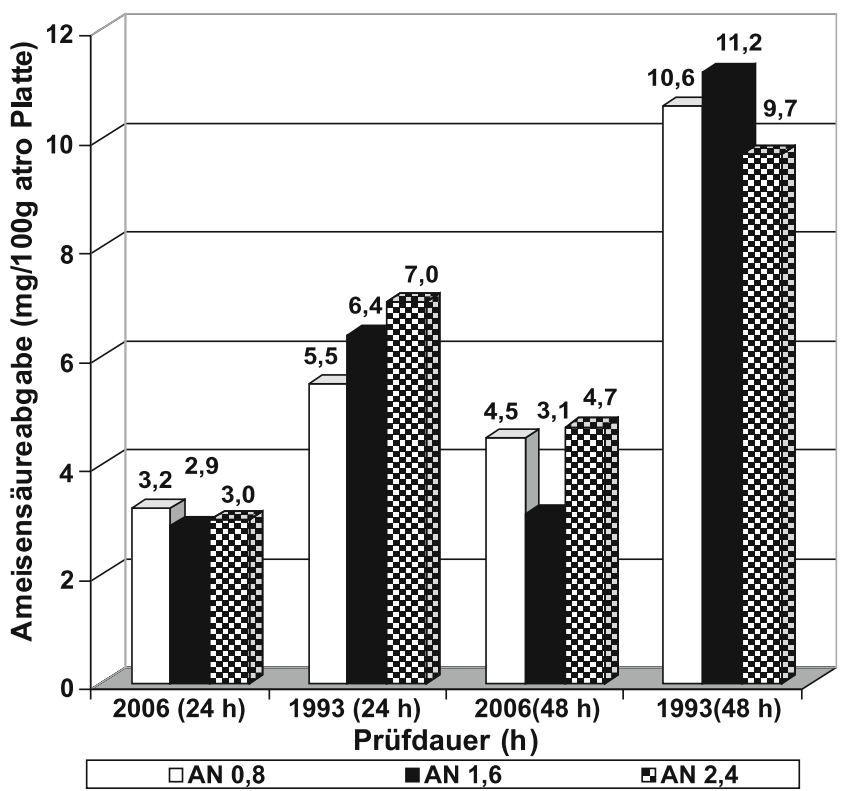

Abb. 1 Ameisensäureabgabe nach 24 und $48 \mathrm{~h}$, gemessen nach der WKI-Flaschen-Methode, von UF-Harz gebundenen Kiefernholzspanplatten unter Einsatz von Ammoniumnitrat als Härtungsbeschleuniger Fig. 1 The release of formic acid after 24 and $48 \mathrm{~h}$ measured by the WKI-method from particleboards bonded with UF-resins using ammonium nitrate as a catalyst

(AN: Ammoniumnitrat)

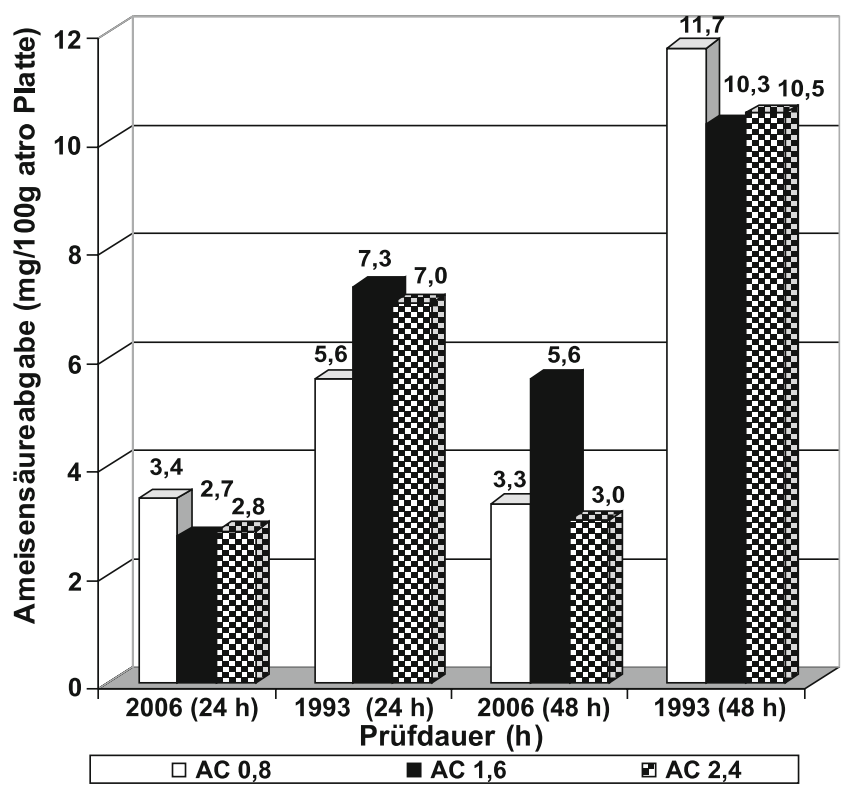

Abb. 2 Ameisensäureabgabe nach 24 und $48 \mathrm{~h}$, gemessen nach der WKI-Flaschen-Methode, von UF-Harz gebundenen Kiefernholzspanplatten unter Einsatz von Ammoniumchlorid als Härtungsbeschleuniger Fig. 2 The release of formic acid after 24 and $48 \mathrm{~h}$ measured by the WKI-method from particleboards bonded with UF-resins using ammonium chloride as a catalyst (AC: Ammoniumchlorid)

abgabe der Platten oder der Zersetzung des UF-Harzes in Zusammenhang steht, muss späteren Untersuchungen vorbehalten bleiben. Hingegen nahmen die Abgabewerte

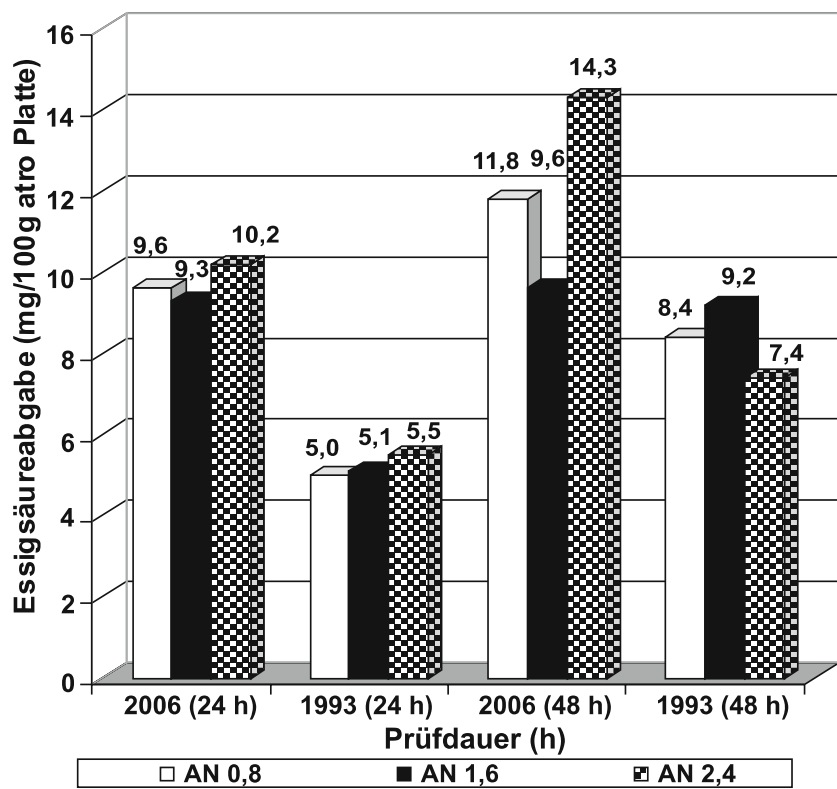

Abb. 3 Essigsäureabgabe, gemessen nach der WKI-Flaschen-Methode, von UF-Harz gebundenen Kiefernholzspanplatten unter Einsatz von Ammoniumnitrat als Härtungsbeschleuniger

Fig. 3 Release of acetic acid measured by the WKI-method from particleboards bonded with UF-resins using ammonium nitrate as a catalyst

(AN: Ammoniumnitrat)

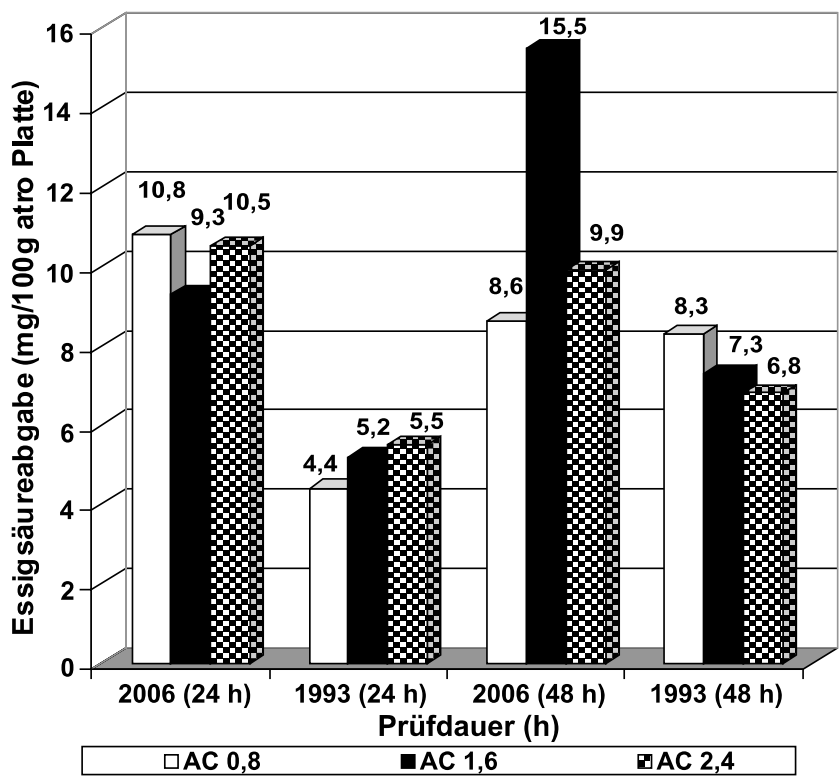

Abb. 4 Essigsäureabgabe, gemessen nach der WKI-Flaschen-Methode, von UF-Harz gebundenen Kiefernholzspanplatten unter Einsatz von Ammoniumchlorid als Härtungsbeschleuniger

Fig. 4 Release of acetic acid measured by the WKI-method from particleboards bonded with UF-resins using ammonium chloride as a catalyst

(AC: Ammoniumchlorid)

an Essigsäure durch die 13-jährige Lagerung zu. Hier ist festzustellen, dass sowohl die nach einer Prüfdauer von $24 \mathrm{~h}$ als auch von $48 \mathrm{~h}$ nach der Flaschenmethode er- 
mittelten Abgabewerte an Essigsäure deutlich zunahmen. Auch hier konnte kein eindeutiger Einfluss des zugesetzten Härters festgestellt werden. Ebenso scheint die Menge des eingesetzten Härters diesen Vorgang nicht entscheidend $\mathrm{zu}$ beeinflussen. Die Zunahme der abgegebenen Menge an Essigsäure, die ausschließlich aus dem Holz stammt, steht in grundsätzlicher Übereinstimmung mit den Untersuchungsergebnissen von Packman (1960), wonach Hölzer unter Einwirkung von Feuchtigkeit und Temperatur je nach Holzart unterschiedliche mit der Lagerung zunehmende Mengen an Säuren freisetzen. Inwieweit die Zunahme der Essigsäureabgabe aus Holzspanplatten auf eine durch den Säuregehalt in Holzspan- und Faserplatten verursachte hydrolytische Wirkung auf das Holz bzw. auf die Holzspäne zurückzuführen ist, dürfte bis lang nicht untersucht worden sein.

Open Access Dieser Artikel wird zu den Bedingungen der "Creative Commons Attribution Noncommercial License" zur Verfügung gestellt. Damit ist eine nichtkommerzielle Nutzung, Verbreitung und Vervielfältigung erlaubt, sofern die Autoren des Artikels und die genaue Quelle angegeben sind.

\section{Literatur}

Çolakoğlu G, Roffael E (1991) Untersuchungen an 40 Jahre alten UFSpanplatten. Adhäsion 35(125):38-39

Emery J A (1986) Formaldehyde Release from Wood Panel Products bonded with Phenol-Formaldehyde Adhesives. In: Formaldehyde Release from Wood Products. ACS Sym Ser 316:26-39

Hameed M, Kraft R, Roffael E (2006) Freier Harnstoff in alten Spanplatten nachweisbar. Holz-Zentralblatt 132:1320-1321

Lee TW, Roffael E, Dix B, Miertzsch H (1994) Influence of different catalyst systems on the Hydrolytic stability of particleboards bonded with unmodified and modified UF-resins. Holzforschung 48:101-106

Packman DF (1960) The Acidity of wood. Holzforschung 14:178-183

Petersen H, Reuther W, Eisele W, Wittman O (1972) Zur Formaldehydabspaltung bei der Spanplattenerzeugung mit HarnstoffFormaldehyd-Bindemitteln. 1. Mitteilung. Holz Roh- Werkst 30: 429-436

Roffael E (1982) Die Formaldehydabgabe von Spanplatten und anderen Holzwerkstoffen. DRW-Verlag, Stuttgart

Roffael E, Dix B, Buchholzer P (1988) Untersuchungen über den Einfluß des Alkalis in PF-Harzen. Adhäsion 32(12):21-29

Roffael E (1989) Zur Bildung von flüchtigen organischen Säuren aus Holzspänen und Holzspanplatten. Holz-Zentralblatt 115:112, $114-115$

Sundin B, Roffael E (1989) Einfluss der Alterung auf die Formaldehydemission von UF-Spanplatten. Holz-Zentralblatt 115(47):704 